\title{
Dietary inflammatory index, cardiometabolic conditions and depression in the Seguimiento Universidad de Navarra cohort study
}

\author{
Almudena Sánchez-Villegas ${ }^{1,2 *}$, Miguel Ruíz-Canela ${ }^{2,3}$, Carmen de la Fuente-Arrillaga ${ }^{2,3}$, Alfredo Gea ${ }^{2,3}$, \\ Nitin Shivappa ${ }^{4,5}$, James R. Hébert ${ }^{4,5}$ and Miguel A. Martínez-González ${ }^{2,3}$ \\ ${ }^{1}$ Nutrition Research Group, Research Institute of Biomedical and Health Sciences, University of Las Palmas de Gran Canaria, \\ CP 35016 Las Palmas de Gran Canaria, Spain \\ ${ }^{2}$ Ciber de Fisiopatología de la Obesidad y Nutrición (CIBER OBN), Instituto de Salud Carlos III, CP 28029 Madrid, Spain \\ ${ }^{3}$ Department of Preventive Medicine and Public Health, University of Navarra, CP 31008 Pamplona, Spain \\ ${ }^{4}$ Cancer Prevention and Control Program, University of South Carolina, Columbia, SC 29208, USA \\ ${ }^{5}$ Department of Epidemiology and Biostatistics, Arnold School of Public Health, University of South Carolina, Columbia, \\ SC 29208, USA \\ (Submitted 9 March 2015 - Final revision received 24 June 2015 - Accepted 16 July 2015 - First published online 7 September 2015)
}

\begin{abstract}
Only one prospective study has analysed the relationship between the inflammatory properties of diet and risk of depression thus far. The aim of this study was to assess the association between the dietary inflammatory index (DII) and the incidence of depression. In a cohort study of 15093 university graduates, participants completed a validated FFQ at baseline and after 10 years of follow-up. The DII was calculated based on the FFQ. Each of the twenty-eight nutrients or foods received a score based on findings from the peer-reviewed literature reporting on the relationships between diet and inflammatory biomarkers (IL-1 $\beta$, IL-4, IL-6, IL-10, TNF- $\alpha$ and C-reactive protein). Participants were classified as having depression if they reported a new clinical diagnosis of depression by a physician, antidepressant drugs, or both. Multivariable Cox regression models were used to estimate hazard ratios (HR) of depression according to quintiles of the DII. After a median 8.5 years of follow-up, we observed 1051 incident cases of depression. The HR for participants in the highest quintile of DII (strongly pro-inflammatory) was $1.47(95 \%$ CI $1.17,1.85)$ compared with those in the bottom quintile, with a significant dose-response relationship $\left(P_{\text {trend }}=0 \cdot 01\right)$. In the subgroup analyses, the association between DII and depression was stronger among participants $>55$ years and among those with cardiometabolic comorbidities (HR 2.70; $95 \%$ CI 1.22, 5.97 and HR 1.80; $95 \%$ CI $1 \cdot 27,2 \cdot 57$, respectively). A pro-inflammatory diet was associated with a significantly higher risk of depression in a Mediterranean population. This association was stronger among older subjects and subjects with cardiometabolic diseases.
\end{abstract}

Key words: Cohort studies: Dietary patterns: Depression: Anti-inflammation: Diabetes

Unipolar depression affects $>151$ million people worldwide and it is projected to be the leading cause of disability-adjusted life years lost in $2030^{(1)}$. Depression shares common mechanisms with obesity, metabolic syndrome (MetS), type 2 diabetes (DM2) and CVD. In fact, the comorbidity of depression with cardiovascular risk factors is frequent ${ }^{(2-4)}$. Metabolic and inflammatory processes, such as reduced insulin sensitivity, elevations in plasma homocysteine levels and, perhaps more importantly, increased production of pro-inflammatory cytokines and endothelial dysfunction, seem to be the major factors responsible for the link between depression and cardiometabolic disorders ${ }^{(5-7)}$.

In general, prospective cohort studies that have analysed the association between dietary patterns and risk of depression have found an inverse association with depression for diets rich in fruits, vegetables, olive oil, legumes and other food items with an anti-inflammatory effect ${ }^{(8-11)}$. In contrast, increased risks have been observed for 'pro-inflammatory' dietary patterns ${ }^{(11,12)}$. However, to our knowledge, only one cohort study, the Nurses' Health Study, has analysed the role of an inflammatory dietary pattern on the risk of depression ${ }^{(13)}$. Our aim was to assess whether a more pro-inflammatory dietary pattern increased the risk of depression in a population from Southern Europe where dietary patterns are substantially different from those followed in the USA. In addition, we aimed to determine whether the association between the dietary inflammatory index (DII) and depression risk was modulated by the cardiometabolic status of the participants.

Abbreviations: DII, dietary inflammatory index; DM2, type 2 diabetes; HR, hazard ratio; HTA, hypertension; MetS, metabolic syndrome.

* Corresponding author: Dr A. Sánchez-Villegas, fax +34928 453 475, email almudena.sanchez@ulpgc.es 


\section{Methods}

\section{Study population}

The 'Seguimiento Universidad de Navarra' (SUN) Project is a multipurpose Spanish cohort composed of university graduates. Baseline assessment and follow-up information was gathered through postal or web-based questionnaires collected every 2 years. The recruitment of participants started on 21 December 1999. It is a dynamic cohort - that is, recruitment is continuously open. The overall retention in the cohort approaches $90 \%$. Further details about the methodology and characteristics of the participants can be found in previously published reports ${ }^{(14)}$. The study was approved by the Institutional Review Board of the University of Navarra. Voluntary completion of the first questionnaire was considered to imply informed consent.

Through June 2014, 22045 subjects had completed the baseline questionnaire of the SUN project. Subjects who had not completed at least one follow-up questionnaire, who were lost to follow-up, who were outside the pre-defined limits for energy intake $(<3347.2 \mathrm{~kJ} / \mathrm{d}(<800 \mathrm{kcal} / \mathrm{d})$ or $>16736 \mathrm{~kJ} / \mathrm{d}$ $(>4000 \mathrm{kcal} / \mathrm{d})$ in men and $<2092 \mathrm{~kJ}(<500 \mathrm{kcal})$ or $14644 \mathrm{~kJ} / \mathrm{d}$ $\left(>3500 \mathrm{kcal} / \mathrm{d}\right.$ ) in women $\left.{ }^{(15)}\right)$ and those who used antidepressant medication or had reported a previous clinical diagnosis of depression (lifetime prevalence) at baseline, or without date of diagnosis of incident depression ( $n$ 60), were excluded from the analyses. After these exclusions, 15093 participants were finally included in this study (Fig. 1)

\section{Exposure assessment}

Dietary intake was assessed at baseline and after 10 years of follow-up with a validated semi-quantitative $\mathrm{FFQ}^{(16,17)}$.
Nutrient intakes of 136 food items were calculated as frequency multiplied by the nutrient composition of a specified portion size for each food item, using an ad hoc computer programme specifically developed for this aim. A trained dietitian updated the nutrient database using the latest available information included in the food composition tables for Spain.

The dietary inflammatory index. The design and development of the DII has been described elsewhere ${ }^{(18)}$. In brief, the DII is a scoring algorithm based on an extensive review of the literature published from 1950 to 2010, linking 1943 articles focussing on the effect of diet on six inflammatory biomarkers (IL-1 $\beta$, IL-4, IL-6, IL-10, TNF- $\alpha$ and C-reactive protein). A total of forty-five food parameters including various macronutrients, micronutrients, flavonoids and individual food items linked to these inflammatory biomarkers were scored according to whether they increased $(+1)$, decreased $(-1)$ or had no effect (0) on inflammation. An overall food parameter-specific inflammatory effect score was calculated for each food item. This percentile was calculated by first linking the dietary data from a study to the regionally representative world database intake, which is based on actual human consumption in eleven populations from different parts of the world that provided a robust estimate of a mean and standard deviation for each parameter. These then become the multipliers to express an individual's exposure relative to the 'standard global mean' as a $Z$-score. This is achieved by subtracting the 'standard global mean' from the amount reported and dividing this value by the standard deviation. To minimise the effect of 'right skewing', this value is then converted to a centred percentile score. The centred percentile score for each food parameter for each

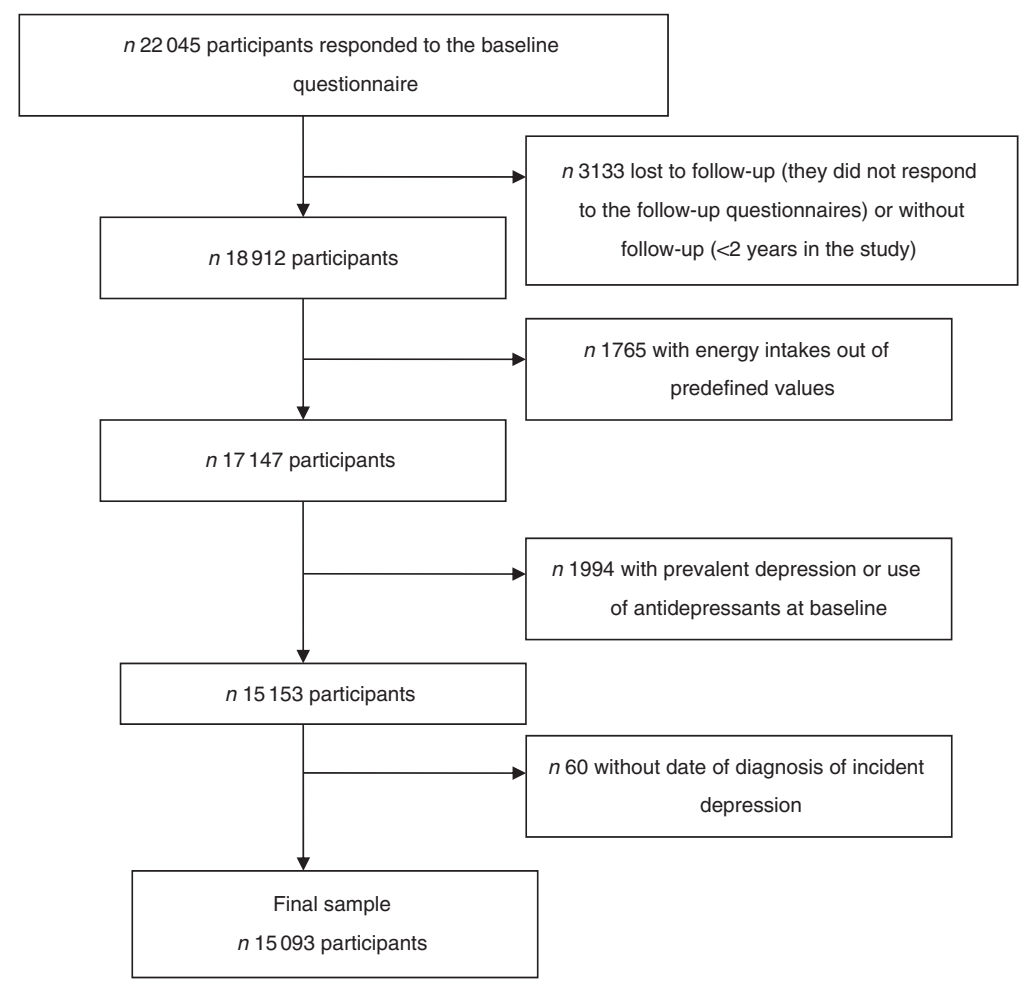

Fig. 1. Flow chart of participants. The Seguimiento Universidad de Navarra Project. 
individual was then multiplied by the respective food parameter effect score, which is derived from the literature review, in order to obtain a food parameter-specific DII score for an individual. All the food parameter-specific DII scores were then summed to create the overall DII score for every participant in the study. The greater the DII score, the more pro-inflammatory the diet. More negative values represent more anti-inflammatory diets. The DII score could take on values ranging from about +8 (maximally pro-inflammatory) to -9 (maximally anti-inflammatory).

Construct validation of the DII was performed using data derived from two different sources of dietary intake information and serum high-sensitivity C-reactive protein as the construct validator $^{(18)}$. Thus far, the DII has been found to be associated with inflammatory cytokines including C-reactive protein and IL-6 $6^{(19-21)}$, the glucose intolerance component of $\mathrm{MetS}^{(20)}$, increased odds of asthma and reduced forced expiratory volume in $1 \mathrm{~s}$ in an Australian population ${ }^{(21)}$, shift work ${ }^{(22)}$, colorectal cancer among women in the Iowa Women's Health Study ${ }^{(23)}$, prostate cancer $^{(24)}$ and pancreatic cancer ${ }^{(25)}$.

In this study, a total of twenty-eight food parameters were available from the FFQ, and therefore could be used to calculate DII (energy, carbohydrate, protein, total fat, alcohol, fibre, cholesterol, SFA, MUFA, PUFA, $n-3, n-6$, trans-fat, niacin, thiamin, riboflavin, vitamin $\mathrm{B}_{12}$, vitamin $\mathrm{B}_{6}, \mathrm{Fe}, \mathrm{Mg}$, Se, $\mathrm{Zn}$, vitamin $\mathrm{A}$, vitamin $\mathrm{C}$, vitamin $\mathrm{D}$, vitamin $\mathrm{E}$, folic acid and caffeine.) The literature-derived inflammatory effect scores assigned to each of the food parameters are shown in Table 1.

\section{Outcome assessment}

Incident cases of depression were defined as participants who positively responded to the following question 'Have you ever been diagnosed of depression by a medical doctor?' or who reported the habitual use of antidepressant drugs in any of the biennial follow-up questionnaires (Q_2-Q_14). Although antidepressants could have been prescribed for conditions other than depression, this situation is highly unusual in Spain. Therefore, we considered both, the use of antidepressants and/or physician diagnosis as depression criteria.

A self-reported physician-provided diagnosis of depression has demonstrated acceptable validity in a subsample of our cohort using the Structured Clinical Interview for DSM-IV as 'gold standard' applied by experienced psychiatrists blinded to the answers of the questionnaires ${ }^{(26)}$. The percentage of confirmed depression was $74.2 \%$ (95\% CI $63.3,85.1)$. The percentage of confirmed non-depression was $81 \cdot 1 \%$ (95\% CI 69.1, 92.9).

\section{Other covariate assessment}

Information about socio-demographic (e.g. sex, age, marital status and employment status) and lifestyle-related variables (e.g. smoking status, physical activity and use of vitamin supplements) were obtained from the baseline questionnaire (Q_0). Physical activity was assessed using a validated physical activity questionnaire with data about seventeen activities ${ }^{(27)}$. Leisure-time activities were computed by assigning a metabolic equivalent score to each activity, multiplied by the time spent for each activity and summing up all activities. A participant was
Table 1. Scoring for each food parameter used for dietary inflammatory index calculation

\begin{tabular}{|c|c|}
\hline Food parameter & Inflammatory effect score* \\
\hline Energy & 0.180 \\
\hline Carbohydrate & 0.097 \\
\hline Fat & 0.298 \\
\hline Alcohol & -0.278 \\
\hline Fibre & -0.663 \\
\hline Protein & 0.021 \\
\hline Vitamin $B_{12}$ & 0.106 \\
\hline Vitamin $\mathrm{B}_{6}$ & -0.365 \\
\hline$\beta$ Carotene & -0.584 \\
\hline$n-3$ & -0.436 \\
\hline$n-6$ & -0.159 \\
\hline MUFA & -0.009 \\
\hline SFA & 0.373 \\
\hline Trans-fat & 0.229 \\
\hline $\mathrm{Fe}$ & 0.032 \\
\hline PUFA & -0.337 \\
\hline Riboflavin & -0.068 \\
\hline Thiamin & -0.098 \\
\hline Niacin & -0.246 \\
\hline Vitamin A & -0.401 \\
\hline $\mathrm{Mg}$ & -0.484 \\
\hline $\mathrm{Zn}$ & -0.313 \\
\hline $\mathrm{Se}$ & $-0 \cdot 191$ \\
\hline Vitamin C & -0.424 \\
\hline Vitamin D & -0.446 \\
\hline Vitamin $\mathrm{E}$ & -0.419 \\
\hline Folate & $-0 \cdot 190$ \\
\hline Caffeine & $-0 \cdot 110$ \\
\hline
\end{tabular}

${ }^{*}$ A negative value indicates anti-inflammatory effect and a positive score indicates pro-inflammatory effect.

considered as a user of vitamin supplements if he/she reported at least the consumption of one of the following vitamin supplements: $\mathrm{A}, \mathrm{B}_{1}, \mathrm{~B}_{2}, \mathrm{~B}_{3}, \mathrm{~B}_{6}, \mathrm{~B}_{9}, \mathrm{~B}_{12}, \mathrm{C}, \mathrm{D}$ or $\mathrm{E}$.

BMI was calculated as weight $(\mathrm{kg})$ divided by the square of height $\left(\mathrm{m}^{2}\right)$ using data collected at baseline and after 10 years of follow-up.

The prevalence and history of CVD, cancer obesity, dyslipidaemia, hypertension (HTA) and DM2 was ascertained at baseline and updated until the end of follow-up or until depression diagnosis was reported. CVD included myocardial infarction, stroke, atrial fibrillation, paroxysmal tachycardia, coronary artery bypass grafting or other re-vascularisation procedures, heart failure, aortic aneurism, pulmonary embolism or peripheral venous thrombosis. All the diagnoses were based on participants self-reporting. The validity of self-reported obesity, dyslipidaemia and HTA diagnoses has been assessed in different subsamples of the cohort ${ }^{(28-30)}$. Self-reported cardiovascular events, cancer and DM2 have been confirmed by medical record review.

Energy and alcohol intake were calculated from the baseline FFQ and after 10 years of follow-up.

\section{Statistical methods}

For each participant, we computed person-years of follow-up from the date of returning the baseline questionnaire to the date of depression diagnosis, the date of death or the date of returning the last follow-up questionnaire, whichever came first. 
Cox regression models (proportional hazards models) were fitted to assess the relationship between the adherence to the DII and the incidence of depression. Hazard ratios (HR) and their $95 \%$ CI were calculated considering the lowest quintile of DII (more anti-inflammatory) as the reference category. To control for potential confounding factors, successive degrees of adjustment were used: (1) in model 1, we adjusted for sex and age (years, continuous); (2) in model 2, we additionally adjusted for BMI $\left(\mathrm{kg} / \mathrm{m}^{2}\right.$, continuous), smoking (non-smoker, ex-smoker, current smoker, missing), physical activity during leisure time (quintiles), use of vitamin supplements and total energy intake $(\mathrm{kJ} / \mathrm{d}(\mathrm{kcal} / \mathrm{d})$, continuous); and, finally, (3) in model 3, we additionally adjusted for the presence of several diseases at baseline (CVD, DM2, HTA and dyslipidaemia). An indicator variable for missing responses was created for smoking. Additional adjustments for cancer history, marital status, unemployment, alcohol intake and menopause status within women were also performed.

Tests of linear trend across increasing quintiles of adherence were conducted by assigning the medians to each quintile and treating it as a continuous variable.

To minimise any effect of a variation in diet, we also calculated the average of DII using an updated DII score with dietary data collected after 10 years of follow-up. To increase accuracy, energy intake and BMI also were updated with the information (Mean values and standard deviations; percentages) obtained after 10 years of follow-up. The prevalence of diseases was updated using the information containing in any of the follow-up questionnaires.

Sensitivity analyses were performed by changing several parameters: (1) adopting different allowed limits for total energy intake; (2) excluding participants with long follow-up ( $\geq 6$ years); (3) excluding early cases (diagnosed during the 1st year of follow-up); (4) excluding participants with special diets (i.e. hypoenergetic, hyperproteic and gluten-free); and HRs were estimated comparing quintiles of the DII in the fully adjusted model.

In addition, subgroup analyses were performed by sex, age group and the presence of several diseases as stratification variables. To assess a possible interaction between DII and sex, age group ( $\leq 55 v .>55$ years), obesity, DM2, CVD and a composite of CVD and CVD risk factors (obesity, DM2, HTA or dyslipidaemia) and product terms were introduced in the different multivariable models. $P$ values for the interaction were calculated using the log-likelihood ratio test.

\section{Results}

We recorded 1051 incident cases of depression during a median follow-up time of 8.5 years. Table 2 shows the distribution of

Table 2. Characteristics of participants according to quintiles of the dietary inflammatory index

\begin{tabular}{|c|c|c|c|c|c|c|}
\hline & $\begin{array}{c}\text { Quintile 1: most } \\
\text { anti-inflammatory ( } n \text { 3019) }\end{array}$ & $\begin{array}{c}\text { Quintile } 2 \\
\text { (n 3019) }\end{array}$ & $\begin{array}{l}\text { Quintile } 3 \\
\text { (n 3018) }\end{array}$ & $\begin{array}{l}\text { Quintile } 4 \\
\text { (n 3019) }\end{array}$ & $\begin{array}{l}\text { Quintile 5: most } \\
\text { pro-inflammatory ( } n \text { 3018) }\end{array}$ & $P$ \\
\hline Age (years) & & & & & & $<0.001$ \\
\hline Mean & $40 \cdot 6$ & 38.9 & $38 \cdot 0$ & $37 \cdot 2$ & $36 \cdot 7$ & \\
\hline SD & $12 \cdot 9$ & $12 \cdot 2$ & $11 \cdot 8$ & 11.5 & 11.4 & \\
\hline Male (\%) & 41.6 & $36 \cdot 8$ & $37 \cdot 0$ & $42 \cdot 9$ & $48 \cdot 5$ & $<0.001$ \\
\hline Married (\%) & $53 \cdot 0$ & $53 \cdot 0$ & 52.5 & $52 \cdot 5$ & $49 \cdot 3$ & 0.019 \\
\hline Unemployment (\%) & 3.5 & $4 \cdot 2$ & $3 \cdot 8$ & $4 \cdot 1$ & $4 \cdot 1$ & 0.557 \\
\hline Smoking status (\%) & & & & & & $<0.001$ \\
\hline Ex-smoker & $33 \cdot 0$ & $29 \cdot 8$ & $30 \cdot 0$ & $29 \cdot 5$ & $27 \cdot 1$ & \\
\hline Current smoker & $17 \cdot 8$ & $20 \cdot 3$ & $20 \cdot 6$ & $23 \cdot 3$ & $25 \cdot 8$ & \\
\hline Vitamin supplements' use* (\%) & 1.9 & $2 \cdot 2$ & 2.5 & 1.9 & $1 \cdot 8$ & 0.339 \\
\hline Special diet at baseline (\%) & $10 \cdot 3$ & $8 \cdot 8$ & $7 \cdot 6$ & $6 \cdot 8$ & $5 \cdot 3$ & $<0.001$ \\
\hline Post-menopause† (\%) & $15 \cdot 3$ & $13 \cdot 6$ & $11 \cdot 1$ & 8.8 & 6.4 & $<0.001$ \\
\hline \multicolumn{7}{|l|}{ Prevalence of diseases (\%) } \\
\hline CVD & $5 \cdot 1$ & $4 \cdot 1$ & $4 \cdot 6$ & 3.9 & $3 \cdot 3$ & 0.004 \\
\hline Cancer & 4.4 & $3 \cdot 1$ & 3.9 & $3 \cdot 2$ & 3.5 & 0.030 \\
\hline DM2 & 2.4 & $2 \cdot 2$ & 1.4 & 1.4 & 1.0 & $<0.001$ \\
\hline HTA & 9.0 & $7 \cdot 3$ & $6 \cdot 2$ & $6 \cdot 0$ & $7 \cdot 0$ & $<0.001$ \\
\hline Dyslipidaemia & 21.5 & $18 \cdot 2$ & $17 \cdot 1$ & $17 \cdot 2$ & $16 \cdot 8$ & $<0.001$ \\
\hline Obesity & $4 \cdot 3$ & 4.8 & $4 \cdot 2$ & 4.5 & $5 \cdot 1$ & 0.456 \\
\hline $\mathrm{BMI}\left(\mathrm{kg} / \mathrm{m}^{2}\right)$ & & & & & & 0.04 \\
\hline Mean & $23 \cdot 6$ & 23.4 & 23.4 & 23.6 & 23.6 & \\
\hline SD & 3.4 & 3.5 & 3.4 & 3.4 & 3.6 & \\
\hline Total energy intake (kJ/d) & & & & & & $<0.001$ \\
\hline Mean & $11497 \cdot 6$ & $10669 \cdot 2$ & 10024.9 & 9175.5 & $7815 \cdot 7$ & \\
\hline $\mathrm{SD}$ & 2397.4 & $2355 \cdot 6$ & $2209 \cdot 2$ & 2092 & 2154.8 & \\
\hline Total energy intake (kcal/d) & & & & & & $<0.001$ \\
\hline Mean & 2748 & 2550 & 2396 & 2193 & 1868 & \\
\hline $\mathrm{SD}$ & 573 & 563 & 528 & 500 & 515 & \\
\hline Physical activity during leisure time (METs-h/week) & & & & & & $<0.001$ \\
\hline Mean & $26 \cdot 7$ & $23 \cdot 2$ & 21.4 & $20 \cdot 1$ & $17 \cdot 7$ & \\
\hline SD & $27 \cdot 1$ & $24 \cdot 2$ & $21 \cdot 3$ & $20 \cdot 6$ & $18 \cdot 9$ & \\
\hline
\end{tabular}

DM2, type 2 diabetes; HTA, hypertension; METs, metabolic equivalents.

* Use of at least one of the following vitamin supplements: $A, B_{1}, B_{2}, B_{3}, B_{6}$, folic acid, $B_{12}, C, D$ or $E$.

† A total of 8847 women were included. 
the baseline characteristics of the participants according to the baseline DII categorised in quintiles. Participants with the highest DII were more likely to be men, single and younger and showed lower prevalence of CVD, DM2 or dyslipidaemia and lower daily energy intake. Some unhealthy lifestyle characteristics such as smoking behaviour and physical inactivity during leisure time were also more prevalent among participants with a more pro-inflammatory diet.

The association between the DII and the risk of depression is shown in Table 3. A higher risk was found for the highest DII in the three models. In model 3 (adjusted for lifestyle factors and the presence of chronic diseases), a higher DII scores (fourth and fifth quintiles) compared with the lowest quintile of DII was associated with an approximately $25-35 \%$ higher risk of depression (multiple-adjusted HR 1.24; $95 \%$ CI 1.00, 1.53 for the fourth quintile and HR 1.37; $95 \%$ CI 1.09, 1.73 for the fifth quintile). Moreover, a significant dose-response relationship was found $\left(P_{\text {trend }}=0 \cdot 015\right)$. When DII was updated using the information collected after 10 years of follow-up, the magnitude of the association was even higher. A relative increment in the risk of depression of approximately $50 \%$ for the comparison between extreme quintiles of DII (HR 1.47; $95 \%$ CI 1.17, 1.85; $\left.P_{\text {trend }}=0.010\right)$ was found. Additional adjustment for personal cancer history, marital status, alcohol intake and unemployment in the overall sample or for menopause status within women did not change the reported associations (data not shown).

Table 4 shows the adjusted HRs in the sensitivity analyses after modifying some of our assumptions. The reported results did not change when the analyses were restricted to those participants with $<6$ years of follow-up. Participants in the highest quintile of the DII had a $47 \%$ higher relative risk of developing depression during the first 6 years of follow-up than participants in the lowest quintile. However, when the analyses were restricted to those participants with a depression diagnosis

Table 3. Risk of incident depression according to the adherence to quintiles of the dietary inflammatory index (DII) (Hazard ratios (HR) and $95 \%$ confidence intervals)

\begin{tabular}{|c|c|c|c|c|c|c|c|c|c|c|c|}
\hline & \multicolumn{2}{|c|}{$\begin{array}{l}\text { Quintile 1: most } \\
\text { anti-inflammatory }\end{array}$} & \multicolumn{2}{|c|}{ Quintile 2} & \multicolumn{2}{|c|}{ Quintile 3} & \multicolumn{2}{|c|}{ Quintile 4} & \multicolumn{2}{|c|}{$\begin{array}{l}\text { Quintile 5: most } \\
\text { pro-inflammatory }\end{array}$} & \multirow[b]{2}{*}{$P_{\text {trend }}$} \\
\hline & $\mathrm{HR}$ & $95 \% \mathrm{Cl}$ & $\mathrm{HR}$ & $95 \% \mathrm{Cl}$ & $\mathrm{HR}$ & $95 \% \mathrm{Cl}$ & $\mathrm{HR}$ & $95 \% \mathrm{Cl}$ & $\mathrm{HR}$ & $95 \% \mathrm{Cl}$ & \\
\hline Median DII & \multicolumn{2}{|c|}{$-3 \cdot 16$} & \multicolumn{2}{|c|}{$-2 \cdot 38$} & \multicolumn{2}{|c|}{$-1 \cdot 75$} & \multicolumn{2}{|c|}{-0.94} & \multicolumn{2}{|c|}{0.66} & \\
\hline Cases & \multicolumn{2}{|c|}{$\begin{array}{r}185 \\
25594\end{array}$} & \multirow{2}{*}{\multicolumn{2}{|c|}{$\begin{array}{r}227 \\
26487\end{array}$}} & \multicolumn{2}{|c|}{211} & \multicolumn{2}{|c|}{211} & \multicolumn{2}{|c|}{217} & \\
\hline Person-years & \multicolumn{2}{|c|}{25594} & & & \multicolumn{2}{|c|}{26143} & \multicolumn{2}{|c|}{26437} & \multicolumn{2}{|c|}{26586} & \\
\hline Crude rates $/ 10^{3 *}$ & $7 \cdot 2$ & $6 \cdot 2,8 \cdot 3$ & $8 \cdot 6$ & $7 \cdot 5,9.8$ & $8 \cdot 1$ & $7 \cdot 0,9 \cdot 2$ & $8 \cdot 0$ & $6 \cdot 9,9 \cdot 1$ & $8 \cdot 2$ & $7 \cdot 2,9 \cdot 3$ & \\
\hline \multicolumn{12}{|l|}{ Model 1† } \\
\hline Baseline DII & 1 & Ref. & $1 \cdot 19$ & $0.98,1.44$ & $1 \cdot 12$ & $0.92,1.37$ & 1.15 & $0.94,1.40$ & $1 \cdot 22$ & $1.00,1.48$ & 0.12 \\
\hline Repeated measures & 1 & Ref. & 1.22 & $1.00,1.48$ & $1 \cdot 15$ & $0.94,1.40$ & 1.27 & $1.04,1.55$ & 1.29 & $1.06,1.58$ & 0.269 \\
\hline \multicolumn{12}{|l|}{ Model $2 \ddagger$} \\
\hline Baseline DII & 1 & Ref. & $1 \cdot 20$ & $0.99,1.46$ & $1 \cdot 16$ & $0.95,1.42$ & $1 \cdot 22$ & $0.99,1.51$ & 1.35 & $1.07,1.71$ & 0.022 \\
\hline Repeated measures & 1 & Ref. & 1.25 & $1.03,1.52$ & $1 \cdot 21$ & $0.99,1.48$ & 1.38 & $1 \cdot 11,1 \cdot 70$ & 1.48 & $1.18,1.86$ & 0.008 \\
\hline \multicolumn{12}{|l|}{ Model $3 \S$} \\
\hline Baseline DII & 1 & Ref. & $1 \cdot 21$ & $0.99,1.47$ & $1 \cdot 17$ & $0.95,1.43$ & 1.24 & $1.00,1.53$ & 1.37 & $1.09,1.73$ & 0.015 \\
\hline Repeated measures & 1 & Ref. & 1.24 & $1.02,1.51$ & $1 \cdot 21$ & $0.98,1.48$ & 1.37 & $1.11,1.69$ & 1.47 & $1.17,1.85$ & 0.010 \\
\hline
\end{tabular}

Ref., referent value.

* Crude rates and $95 \%$ confidence intervals.

† Model 1: adjusted for age and sex. Repeated measures. Cumulative average for DII (at baseline and after 10 years of follow-up). Energy intake, BMI and prevalence of diseases were also updated.

¥ Model 2: this includes all variables from model 1 plus BMI, smoking, physical activity during leisure time, use of vitamin supplements and total energy intake.

$\S$ Model 3: this includes all variables from model 2 with additional adjustment for the presence of CVD, type 2 diabetes, hypertension and dyslipidaemia at baseline.

Table 4. Sensitivity analyses*

(Hazard ratios (HR) and $95 \%$ confidence intervals)

\begin{tabular}{|c|c|c|c|c|c|}
\hline & \multirow[b]{2}{*}{ Cases } & \multirow[b]{2}{*}{$n$} & \multicolumn{2}{|c|}{ Quintile 5 v. quintile 1 (Ref.) } & \multirow[b]{2}{*}{$P_{\text {trend }} t$} \\
\hline & & & $\mathrm{HR}$ & $95 \% \mathrm{Cl}$ & \\
\hline Energy limits: 5 th to 95 th percentiles $\ddagger$ & 1067 & 15043 & 1.41 & $1 \cdot 11,1.80$ & 0.008 \\
\hline Energy limits: 1st to 99th percentiles§ & 1159 & 16338 & 1.43 & $1 \cdot 13,1.81$ & 0.005 \\
\hline Excluding participants with $\geq 6$ years of follow-up & 685 & 3156 & 1.47 & $1.11,1.95$ & 0.005 \\
\hline Excluding cases diagnosed during the first 2 years of follow-up & 868 & 14910 & $1 \cdot 27$ & $0.98,1.64$ & $0 \cdot 14$ \\
\hline Excluding cases diagnosed during the first 3 years of follow-up & 777 & 14819 & 1.28 & $0.97,1.69$ & $0 \cdot 16$ \\
\hline Excluding subjects with an special diet at baseline or without this information & 909 & 13587 & $1 \cdot 29$ & $1.00,1.66$ & 0.07 \\
\hline
\end{tabular}

Ref., referent value

* The association between extreme quintiles of adherence to the dietary inflammatory index and depression. Adjusted for age, sex, BMI, smoking, physical activity during leisure time, use of vitamin supplements, total energy intake and presence of several diseases at baseline (CVD, diabetes mellitus type 2, hypertension and dyslipidaemia).

$\dagger$ For the five quintiles.

$\ddagger>5648.4 \mathrm{~kJ} / \mathrm{d}(>1350 \mathrm{kcal} / \mathrm{d})$ and $<17405.4 \mathrm{~kJ} / \mathrm{d}(<4160 \mathrm{kcal} / \mathrm{d})$ in men and $>4020.8 \mathrm{~kJ} / \mathrm{d}(>961 \mathrm{kcal} / \mathrm{d})$ and $<16970.3 \mathrm{~kJ} / \mathrm{d}(<4056 \mathrm{kcal} / \mathrm{d})$ in women.

$\S>3711.2 \mathrm{~kJ} / \mathrm{d}(>887 \mathrm{kcal} / \mathrm{d})$ and $<22781.9 \mathrm{~kJ} / \mathrm{d}(<5445 \mathrm{kcal} / \mathrm{d})$ in men and $>5744.6 \mathrm{~kJ} / \mathrm{d}(>1373 \mathrm{kcal} / \mathrm{d})$ and $<23735.8 \mathrm{~kJ} / \mathrm{d}(<5673 \mathrm{kcal} / \mathrm{d})$ in women. 


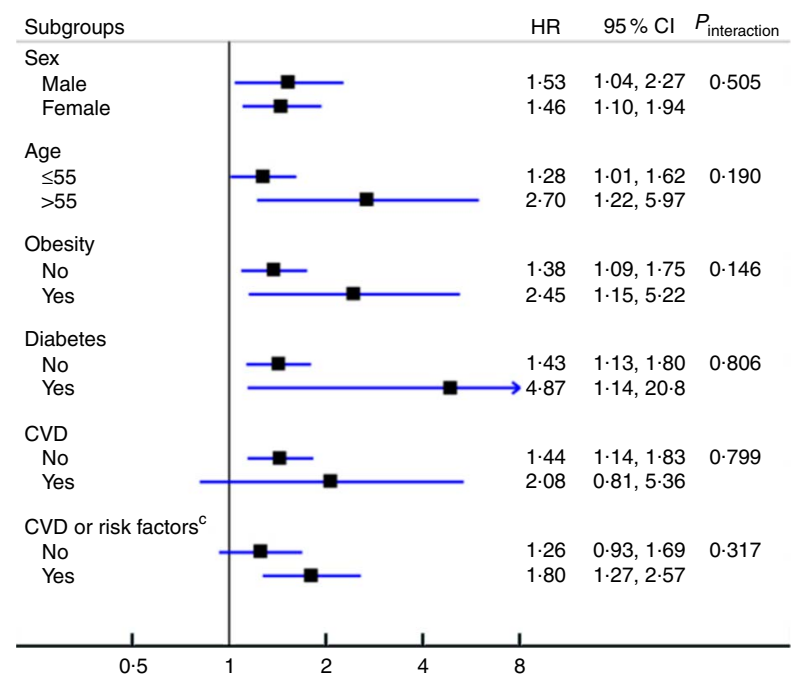

Fig. 2. Subgroups analyses. Hazard ratios $(\mathrm{HR})(95 \% \mathrm{Cl})^{\mathrm{a}}$ for the association between extreme quintiles of adherence to the dietary inflammatory index (DII) and depression. ${ }^{a}$ Adjusted for age, sex, BMl, smoking, physical activity during leisure time, use of vitamin supplements, total energy intake and prevalence of several diseases (CVD, type 2 diabetes, hypertension and dyslipidaemia). ${ }^{\mathrm{b}}$ Repeated measures. Cumulative average for DII (at baseline and after 10 years of follow-up). Energy intake, BMI and prevalence of diseases were also updated. 'Prevalence of obesity, type 2 diabetes, hypertension or dyslipidaemia.

after 2 or 3 years of follow-up, the association was attenuated and it was no longer statistically significant.

The association between the DII score and depression risk according to categories of several baseline characteristics is shown in Fig. 2. Although none of the interaction terms included in the multivariable models were statistically significant, a stronger association with depression was evident when comparing DII quintile 5 to quintile 1 for participants aged $\geq 55$ years (HR 2.70; 95\% CI 1.22, 5.97) and for those with concomitant diseases including obesity (HR 2.45; $95 \%$ CI 1.15, 5.22); DM2 (HR 4.87; $95 \%$ CI 1.14, 20.81); CVD (HR 2.08; $95 \%$ CI 0.81, 5.36); or a composite of cardiometabolic diseases or risks (HR 1.80; $95 \%$ CI $1 \cdot 27,2 \cdot 57$ ).

\section{Discussion}

In this analysis from the SUN cohort study, participants with the highest DII score (representing the most pro-inflammatory dietary potential) showed a $47 \%$ higher risk of developing depression compared with participants with the lowest DII (those consuming diets with the greatest anti-inflammatory potential). This result is consistent with that reported in another prospective cohort study, the Nurses' Health Study, which found a $41 \%$ higher risk for the highest $v$. the lowest quintile of inflammatory properties of the $\operatorname{diet}^{(13)}$. Although it used an identical definition of the outcome, the American cohort used a different approach (reduced rank regression) to classify participants according to the inflammatory potential of their dietary patterns.

The results obtained in our analyses confirm those found in other analyses evaluating the role of diet in depression.
Numerous prospective studies have reported inverse associations between diet quality or the adherence to prudent or traditional diets and the risk of depression with consistent results across different countries and cultures ${ }^{(8-11,31)}$. Similarly, the Mediterranean diet has shown an anti-inflammatory effect $^{(32,33)}$. In contrast, Western dietary patterns, characterised by the consumption of processed foods, have been directly associated with depressive disorders ${ }^{(11,12)}$ as well as with elevated levels of some pro-inflammatory markers ${ }^{(34,35)}$.

A large number of studies have reported the possible role of inflammation in depression through mechanisms such as activation of the hypothalamic-pituitary-adrenal axis, tryptophan depletion and decrease in brain-derived neurotrophic factor availability ${ }^{(36)}$. Cross-sectional evidences from epidemiological studies seem to confirm a bidirectional relationship ${ }^{(37)}$. Although systemic inflammatory markers have been prospectively associated with depression ${ }^{(38,39)}$, not all longitudinal studies have found a significant relationship ${ }^{(40)}$. Thus, the contribution of a pro-inflammatory dietary pattern to the development of depression has not been easy to understand.

One of the most remarkable results obtained in our analysis suggests that the effect of a pro-inflammatory diet (stressor) on depression could be particularly detrimental among individuals with some cardiometabolic conditions (prevalent chronic conditions such as CVD, DM2 or/and obesity) or among those $\geq 55$ years of age. Although the interaction was not statistically significant, its magnitude could be biologically relevant. These results are in accordance with those recently obtained in the PREDIMED trial. In that trial, the adherence to a Mediterranean dietary pattern supplemented with nuts was particularly important to prevent depression among participants with $\mathrm{DM} 2^{(41)}$. Similarly, this hypothesis has been suggested in a cross-sectional study conducted recently in Australia, which found that a healthy dietary pattern was associated with a reduced likelihood of depressive symptoms, especially for those with $\mathrm{DM} 2^{(42)}$.

One possible explanation for our observed results is that the presence of several chronic conditions might lead to maladaptive stress responses within this group, including heightened low-grade inflammation and HPA axis nonhabituation. In fact, cytokine levels are strongly affected by socio-demographic and environmental factors such as age, sex, smoking, exercise, obesity or insulin resistance. The link between CVD, inflammation and depressive disorders has been repeatedly suggested. Over the last few years, several studies have established the possible link between inflammation, depression and not only CVD events but also other related conditions such as DM2, MetS or obesity ${ }^{(5,6,43-45)}$. In fact, hyperleptinaemia or insulin resistance in obesity, MetS and DM2 have been linked to inflammatory processes ${ }^{(46)}$, which also are common in depressive disorders. Similarly, obesity (a pro-inflammatory condition ${ }^{(47)}$ ) has been found to be associated with elevated cortisol secretion and higher HPA axis reactivity to psychological stress as well as physiological and pharmacological stimulation ${ }^{(48)}$. Moreover, in a recent study, McInnis et $a l^{(49)}$ found that individuals with higher measures of adiposity showed less efficient HPA axis habituation as well as sensitisation of IL- 6 responses to repeated acute stress, indicating that increased adiposity would be related to altered 
endocrine and IL-6 stress responses. Consistent with our findings, Grosse et al. ${ }^{(50)}$ reported that monocyte immune activation was not uniformly elevated in all depressive patients, but it was increased only in older subjects. Indeed, the pro-inflammatory effect of the diet could be more relevant among older subjects by inducing sensitisation with increased activation of the inflammatory response system.

Some potential limitations of our study need to be mentioned. Self-reporting of a clinical diagnosis or the use of medication was used as the criteria to establish depression. Our validation study found low sensitivity $(0.37)$ but very high specificity $(0.96)$ for the self-reported diagnosis of depression ${ }^{(26)}$. Theoretically, with perfect specificity, non-differential sensitivity of disease misclassification will not bias the relative risk estimate ${ }^{(51)}$. Similarly, although the validity and reliability of the FFQ have been evaluated ${ }^{(16,17)}$, some degree of misclassification may exist in the dietary assessment. However, the use of a cohort design mitigates this to some extent. In this context, misclassification is more likely to be non-differential, and therefore would bias the results towards the null. Another concern is the potential of reverse causation. Participants with subclinical depression at the beginning of our study might have changed their food habits precisely because of their mood disorder. In fact, when the analyses were restricted to those participants who reported a depression diagnosis after 2 or 3 years of follow-up, the association was attenuated. To avoid the possibility that the induction period for the effect of baseline diet might be shorter than the time of follow-up of these 'late' cases (some of them diagnosed after 14 years of follow-up), we excluded participants with $>6$ years of follow-up from the main analyses, updated nutritional data and used cumulative DII after 10 years of follow-up. Both analyses reported similar results, and with an even higher relative risk for depression associated with high DII scores. Another possible weakness is the inability to control for several potential confounders related to psychological features. Finally, our participants are not representative of the general Spanish population. We restricted our cohort to highly educated participants to obtain a better quality of selfreported information, to improve the retention rate and to minimise confounding by educational level, and therefore by socio-economic status ${ }^{(52)}$.

Several strengths of our study also deserve to be mentioned. They include its large sample size, prospective design, long-term follow-up, the use of updated nutritional data, the ability to control for a variety of major potential confounders, the existence of published validation studies of our assessments and the restriction to highly educated participants who may be able to provide more reliable information.

In conclusion, a higher DII (indicative of a more proinflammatory diet) was associated with an increased risk of developing depression among participants from the SUN cohort study. This effect could be even more important among older individuals and those with prevalent comorbidities related to inflammation such as CVD, DM2 or obesity. Further studies analysing the link between inflammation, depression and cardiometabolic conditions are warranted to deepen our understanding about the role of diet in developing depression and other mental disorders

\section{Acknowledgements}

The authors are indebted to the participants of the SUN Project for their continued co-operation and participation. They also thank the other members of the SUN Group: A. Alonso, M. T. Barrio López, F. J. Basterra-Gortari, S. Benito Corchón, M. Bes-Rastrollo, J. J. Beunza, S. Carlos Chillerón, L. Carmona, S. Cervantes, J. de Irala Estévez, P. A. de la Rosa, M. Delgado Rodríguez, C. L. Donat Vargas, M. Donázar, A. Fernández Montero, C. Galbete Ciáurriz, M. García López, E. Goñi Ochandorena, F. Guillén Grima, A. Hernández, F. Lahortiga, J. Llorca, C. López del Burgo, A. Marí Sanchís, A. Martí del Moral, N. Martín Calvo, J. A. Martínez, J. M. Núñez-Córdoba, A. M. Pimenta, A. Ruiz Zambrana, D. Sánchez Adán, C. Sayón Orea, E. Toledo Atucha, J. Toledo Atucha, Z. Vázquez Ruiz and I. Zazpe García.

The SUN Study has received funding from the Spanish Government (current grants PI10/02658, PI10/02293, PI13/ 00615, PI14/01798, RD06/0045, G03/140 and 87/2010), the Navarra Regional Government (45/2011, 27/2011) and the University of Navarra. A. G. is supported by a FPU fellowship from the Spanish Government. N. S. and J. R. H. were supported by grant number R44DK103377 from the United States National Institute of Diabetes and Digestive and Kidney Diseases.

Funding sources had no role in the design, collection, analysis and interpretation of the data; in the writing; and in the decision to submit the paper for publication.

Study concept and design: A. S.-V. and M. A. M.-G.; acquisition of data: A. S.-V., N. S., J. R. H. and C. de la F.-A.; analysis and interpretation of data: A. S.-V., A. G. and M. A. M.-G.; drafting of the manuscript: A. S.-V. and M. R.-C.; critical revision of the manuscript for important intellectual content: all co-authors.

There are no conflicts of interest to declare.

J. R. H. owns the controlling interest in Connecting Health Innovations LLC (CHI), a company planning to licence the right to his invention of the dietary inflammatory index (DII) from the University of South Carolina in order to develop computer and smart phone applications for patient counselling and dietary intervention in clinical settings. N. S. is an employee of CHI. The subject matter of this paper does not have any direct bearing on that work, nor has the activity exerted any influence on this project.

\section{References}

1. World Health Organization (2008) The Global Burden of Disease 2004 Update. Geneva, Switzerland: WHO.

2. Valkanova V \& Ebmeier KP (2013) Vascular risk factors and depression in later life: a systematic review and meta-analysis. Biol Psychiatry 73, 406-413.

3. Luppino FS, de Wit LM, Bouvy PF, et al. (2010) Overweight, obesity, and depression: a systematic review and metaanalysis of longitudinal studies. Arch Gen Psychiatry 67, 220-229.

4. Pan A, Keum N, Okereke OI, et al. (2012) Bidirectional association between depression and metabolic syndrome: a systematic review and meta-analysis of epidemiological studies. Diabetes Care 35, 1171-1180. 
5. Hood KK, Lawrence JM, Anderson A, et al. (2012) Metabolic and inflammatory links to depression in youth with diabetes. Diabetes Care 35, 2443-2446.

6. Doyle TA, de Groot M, Harris T, et al. (2013) Diabetes, depressive symptoms, and inflammation in older adults: results from the health, aging, and body composition study. J Psychosom Res 75, 419-424.

7. Poole L, Dickens C \& Steptoe A (2011) The puzzle of depression and acute coronary syndrome: reviewing the role of acute inflammation. $J$ Psychosom Res 71, 61-68.

8. Rienks J, Dobson AJ \& Mishra GD (2013) Mediterranean dietary pattern and prevalence and incidence of depressive symptoms in mid-aged women: results from a large communitybased prospective study. Eur J Clin Nutr 67, 75-82.

9. Sánchez-Villegas A, Delgado-Rodríguez M, Alonso A, et al. (2009) Association of the Mediterranean dietary pattern with the incidence of depression: the Seguimiento Universidad de Navarra/University of Navarra follow-up (SUN) cohort. Arch Gen Psychiatry 66, 1090-1098.

10. Jacka FN, Kremer PJ, Berk M, et al. (2011) A prospective study of diet quality and mental health in adolescents. PLOS ONE $\mathbf{6}$, e24805.

11. Akbaraly TN, Brunner EJ, Ferrie JE, et al. (2009) Dietary pattern and depressive symptoms in middle age. $\mathrm{Br} J$ Psychiatry 195, 408-413.

12. Le Port A, Gueguen A, Kesse-Guyot E, et al. (2012) Association between dietary patterns and depressive symptoms over time: a 10-year follow-up study of the GAZEL cohort. PLOS ONE 7, e51593.

13. Lucas M, Chocano-Bedoya P, Shulze MB, et al. (2014) Inflammatory dietary pattern and risk of depression among women. Brain Behav Immun 36, 46-53.

14. Segui-Gomez M, de la Fuente C, Vazquez Z, et al. (2006) Cohort profile: the 'Seguimiento Universidad de Navarra' (SUN) study. Int J Epidemiol 35, 1417-1422.

15. Willett W (1998) Issues in analysis and presentation of dietary data. In Nutritional Epidemiology, 2nd ed. pp 321-346 [Willett WC, editor]. New York: Oxford University Press.

16. Fernandez-Ballart JD, Pinol JL, Zazpe I, et al. (2009) Relative validity of a semi-quantitative food-frequency questionnaire in an elderly Mediterranean population of Spain. Br J Nutr 103, 1808-1816.

17. De la Fuente-Arrillaga C, Vázquez Ruiz Z, Bes-Rastrollo M, et al. (2010) Reproducibility of an FFQ validated in Spain. Public Health Nutr 3, 1364-1372.

18. Shivappa N, Steck SE, Hurley TG, et al. (2014) Designing and developing a literature-derived, population-based dietary inflammatory index. Public Health Nutr 17, 1689-1696.

19. Shivappa N, Steck SE, Hurley TG, et al. (2014) A populationbased dietary inflammatory index predicts levels of C-reactive protein in the Seasonal Variation of Blood Cholesterol Study (SEASONS). Public Health Nutr 17, 1825-1833.

20. Wirth MD, Burch J, Shivappa N, et al. (2014) Association of a dietary inflammatory index with inflammatory indices and metabolic syndrome among police officers. J Occup Environ Med 56, 986-989.

21. Wood L, Shivappa N, Berthon BS, et al. (2015) Dietary inflammatory index is related to asthma risk, lung function and systemic inflammation in asthma. Clin Exp Allergy 45, 177-183.

22. Wirth MD, Burch J, Shivappa N, et al. (2014) Dietary inflammatory index scores differ by shift work status: NHANES 2005 to 2010. J Occup Environ Med 56, 145-148.

23. Shivappa N, Prizment AE, Blair CK, et al. (2014) Dietary inflammatory index (DII) and risk of colorectal cancer in Iowa Women's Health Study. Cancer Epidemiol Biomarkers Prev 23, 2383-2392.
24. Shivappa N, Bosetti C, Zucchetto A, et al. (2015) Association between dietary inflammatory index and prostate cancer among Italian men. BrJ Nutr (in the press).

25. Shivappa N, Bosetti C, Zucchetto A, et al. (2015) Dietary Inflammatory Index and risk of pancreatic cancer in an Italian case-control study. Br J Nutr (in the press).

26. Sanchez-Villegas A, Schlatter J, Ortuno F, et al. (2008) Validity of a self-reported diagnosis of depression among participants in a cohort study using the Structured Clinical Interview for DSM-IV (SCID-I). BMC Psychiatry 8, 43.

27. Martínez-González MA, López-Fontana C, Varo JJ, et al. (2005) Validation of the Spanish version of the physical activity questionnaire used in the Nurses' Health Study and the Health Professionals' follow-up study. Public Health Nutr $\mathbf{8}$, 920-927.

28. Bes-Rastrollo M, Perez JR, Sanchez-Villegas A, et al. (2005) Validation of the self-reported weight and body mass index of the participants in a cohort of university graduates. Rev Esp Obes 3, 352-358.

29. Fernández-Montero A, Beunza JJ, Bes-Rastrollo M, et al. (2011) Validity of self-reported metabolic syndrome components in a cohort study. Gac Sanit 25, 303-307.

30. Alonso A, Beunza JJ, Delgado-Rodríguez M, et al. (2005) Validation of self reported diagnosis of hypertension in a cohort of university graduates in Spain. BMC Public Health $\mathbf{5}, 94$.

31. Lai JS, Hiles S, Bisquera A, et al. (2014) A systematic review and meta-analysis of dietary patterns and depression in community-dwelling adults. Am J Clin Nutr 99, 181-197.

32. Casas R, Sacanella E, Urpí-Sardà M, et al. (2014) The effects of the Mediterranean diet on biomarkers of vascular wall inflammation and plaque vulnerability in subjects with high risk for cardiovascular disease. A randomized trial. PLOS ONE 9, e100084.

33. Chrysohoou C, Panagiotakos DB, Pitsavos C, et al. (2004) Adherence to the Mediterranean diet attenuates inflammation and coagulation process in healthy adults: the ATTICA Study. J Am Coll Cardiol 44, 152-158.

34. Lopez-Garcia E, Schulze MB, Fung TT, et al. (2004) Major dietary patterns are related to plasma concentrations of markers of inflammation and endothelial dysfunction. Am J Clin Nutr 80, 1029-1035.

35. Fung TT, Rimm EB, Spiegelman D, et al. (2001) Association between dietary patterns and plasma biomarkers of obesity and cardiovascular disease risk. Am J Clin Nutr 73, 61-67.

36. Zunszain PA, Hepgul N \& Pariante CM (2013) Inflammation and depression. Curr Top Behav Neurosci 14, 135-151.

37. Howren MB, Lamkin DM \& Suls J (2009) Associations of depression with C-reactive protein, IL-1, and IL-6: a metaanalysis. Psychosom Med 71, 171-186.

38. Khandaker GM, Pearson RM, Zammit S, et al. (2014) Association of serum interleukin 6 and c-reactive protein in childhood with depression and psychosis in young adult life: a population-based longitudinal study. JAMA Psychiatry $\mathbf{7 1}$, 1121-1128.

39. Pasco JA, Nicholson GC, Williams LJ, et al. (2010) Association of high-sensitivity C-reactive protein with de novo major depression. Br J Psychiatry 197, 372-377.

40. Chocano-Bedoya PO, Mirzaei F, O'Reilly EJ, et al. (2014) C-reactive protein, IL-6, soluble tumor necrosis factor $\alpha$ receptor 2 and incident clinical depression. $J$ Affect Disord 163, 25-32.

41. Sánchez-Villegas A, Martínez-González MA, Estruch R, et al. (2013) Mediterranean dietary pattern and depression: the PREDIMED randomized trial. BMC Med 11, 208.

42. Dipnall JF, Pasco JA, Meyer D, et al. (2014) The association between dietary patterns, diabetes and depression. I Affect Disord 174C, 215-224. 
43. Au B, Smith KJ, Gariépy G, et al. (2014) C-reactive protein, depressive symptoms, and risk of diabetes: results from the English Longitudinal Study of Ageing (ELSA). J Psychosom Res 77, 180-186.

44. Schmidt FM, Lichtblau N, Minkwitz J, et al. (2014) Cytokine levels in depressed and non-depressed subjects, and masking effects of obesity. I Psychiatr Res 55, 29-34.

45. Laake JP, Stahl D, Amiel SA, et al. (2014) The association between depressive symptoms and systemic inflammation in people with type 2 diabetes: findings from the South London Diabetes Study. Diabetes Care 37, 2186-2192.

46. Flehmig G, Scholz M, Klöting N, et al. (2014) Identification of adipokine clusters related to parameters of fat mass, insulin sensitivity and inflammation. PLOS ONE 9, e99785.

47. Ouchi N, Parker JL, Lugus JJ, et al. (2011) Adipokines in inflammation and metabolic disease. Nature Rev Immunol 11, 85-97.
48. Bjorntorp P (1993) Visceral obesity: a 'civilization syndrome'. Obes Res 1, 206-222.

49. McInnis CM, Thoma MV, Gianferante D, et al. (2014) Measures of adiposity predict IL- 6 responses to repeated psychosocial stress. Brain Behav Immun 42, 33-40.

50. Grosse L, Carvalho LA, Wijkhuijs AJ, et al. (2014) Clinical characteristics of inflammation-associated depression: Monocyte gene expression is age-related in major depressive disorder. Brain Behav Immun 44, 48-56.

51. Greenland S \& Lash TL (2008) Bias analysis. In Modern Epidemiology, 3rd ed. pp. 359-380 [Rothman KJ, Greenland S and Lash TL, editors]. Philadelphia, PA: Lippincott Williams and Wilkins.

52. Rothman KJ, Greenland S \& Lash TL (2008) Design strategies to improve study accuracy. In Modern Epidemiology, 3rd ed. pp. 168-182 [Rothman KJ, Greenland S and Lash TL, editors]. Philadelphia, PA: Lippincott Williams and Wilkins. 